Kragujevac Journal of Mathematics

Volume 44(2) (2020), Pages 189-203.

\title{
EXISTENCE, UNIQUENESS AND STABILITY OF PERIODIC SOLUTIONS FOR NONLINEAR NEUTRAL DYNAMIC EQUATIONS
}

\author{
F. BOUCHELAGHEM ${ }^{1}$, A. ARDJOUNI ${ }^{2}$, AND A. DJOUDI $^{1}$
}

ABSTRACT. The nonlinear neutral dynamic equation with periodic coefficients

$$
\begin{aligned}
& {[u(t)-g(u(t-\tau(t)))]^{\Delta} } \\
= & p(t)-a(t) u^{\sigma}(t)-a(t) g\left(u^{\sigma}(t-\tau(t))\right)-h(u(t), u(t-\tau(t)))
\end{aligned}
$$

is considered in this work. By using Krasnoselskii's fixed point theorem we obtain the existence of periodic and positive periodic solutions and by contraction mapping principle we obtain the uniqueness. Stability results of this equation are analyzed. The results obtained here extend the work of Mesmouli, Ardjouni and Djoudi [14].

\section{INTRODUCTION}

In 1988, Stephan Hilger [10] introduced the theory of time scales (measure chains) as a means of unifying discrete and continuum calculi. Since Hilger's initial work there has been significant growth in the theory of dynamic equations on time scales, covering a variety of different problems (see $[7,8,13]$ and references therein).

Let $\mathbb{T}$ be a periodic time scale such that $0 \in \mathbb{T}$. In this article, we are interested in the analysis of qualitative theory of periodic and positive periodic solutions of neutral dynamic equations. Motivated by the papers $[1-6,11,12,14,15,17]$ and the references therein, we consider the following nonlinear neutral dynamic equation

$$
\begin{aligned}
& {[u(t)-g(u(t-\tau(t)))]^{\Delta} } \\
= & p(t)-a(t) u^{\sigma}(t)-a(t) g\left(u^{\sigma}(t-\tau(t))\right)-h(u(t), u(t-\tau(t))) .
\end{aligned}
$$

Key words and phrases. Fixed point, periodic solutions, stability, dynamic equations, time scales. 2010 Mathematics Subject Classification. Primary: 34K13, 34A34. Secondary: 34K30, 34L30.

DOI 10.46793/KgJMat2002.189B

Received: April 14, 2017.

Accepted: March 27, 2018. 
Throughout this paper we assume that $a, p$ and $\tau$ are real valued rd-continuous functions with $a$ and $\tau$ are positive functions, $i d-\tau: \mathbb{T} \rightarrow \mathbb{T}$ is increasing so that the function $u(t-\tau(t))$ is well defined over $\mathbb{T}$. The functions $g$ and $h$ are continuous in their respective arguments. To reach our desired end we have to transform (1.1) into an integral equation written as a sum of two mapping, one is a contraction and the other is continuous and compact. After that, we use Krasnoselskii's fixed point theorem, to show the existence of periodic and positive periodic solutions. We also obtain the existence of a unique periodic solution by employing the contraction mapping principle. In addition to the study of existence and uniqueness, in this research we obtain sufficient conditions for the stability of the periodic solution by using the contraction mapping principle.

The organization of this paper is as follows. In Section 2, we introduce some notations and definitions, and state some preliminary material needed in later sections. We will state some facts about the exponential function on a time scale as well as the fixed point theorems. For details on fixed point theorems we refer the reader to [16]. In Section 3, we establish the existence and uniqueness of periodic solutions. In Section 4, we give sufficient conditions to ensure the existence of positive periodic solutions. The stability of the periodic solution is the topic of Section 5. The results presented in this paper extend the main results in [14].

\section{Preliminaries}

A time scale is an arbitrary nonempty closed subset of real numbers. The study of dynamic equations on time scales is a fairly new subject, and research in this area is rapidly growing (see $[1-6,11,12,15]$ and papers therein). The theory of dynamic equations unifies the theories of differential equations and difference equations. We suppose that the reader is familiar with the basic concepts concerning the calculus on time scales for dynamic equations. Otherwise one can find in Bohner and Peterson books $[7,8,13]$ most of the material needed to read this paper. We start by giving some definitions necessary for our work. The notion of periodic time scales is introduced in Kaufmann and Raffoul [11]. The following two definitions are borrowed from [11].

Definition 2.1. We say that a time scale $\mathbb{T}$ is periodic if there exist a $\omega>0$ such that if $t \in \mathbb{T}$ then $t \pm \omega \in \mathbb{T}$. For $\mathbb{T} \neq \mathbb{R}$, the smallest positive $\omega$ is called the period of the time scale.

Example 2.1. The following time scales are periodic.

(a) $\mathbb{T}=\bigcup_{i=-\infty}^{\infty}[2(i-1) h, 2 i h], h>0$, has period $\omega=2 h$.

(b) $\mathbb{T}=h Z$ has period $\omega=h$.

(c) $\mathbb{T}=\mathbb{R}$.

(d) $\mathbb{T}=\left\{t=k-q^{m}: k \in Z, m \in N_{0}\right\}$, where $0<q<1$ has period $\omega=1$.

Remark 2.1 ([11]). All periodic time scales are unbounded above and below. 
Definition 2.2. Let $\mathbb{T} \neq \mathbb{R}$ be a periodic time scale with period $\omega$. We say that the function $f: \mathbb{T} \rightarrow \mathbb{R}$ is periodic with period $T$ if there exists a natural number $n$ such that $T=n \omega, f(t \pm T)=f(t)$ for all $t \in \mathbb{T}$ and $T$ is the smallest number such that $f(t \pm T)=f(t)$.

If $\mathbb{T}=\mathbb{R}$, we say that $f$ is periodic with period $T>0$ if $T$ is the smallest positive number such that $f(t \pm T)=f(t)$ for all $t \in \mathbb{T}$.

Remark 2.2 ([11]). If $\mathbb{T}$ is a periodic time scale with period $\omega$, then $\sigma(t \pm n \omega)=$ $\sigma(t) \pm n \omega$. Consequently, the graininess function $\mu$ satisfies $\mu(t \pm n \omega)=\sigma(t \pm n \omega)-$ $(t \pm n \omega)=\sigma(t)-t=\mu(t)$ and so, is a periodic function with period $\omega$.

Definition $2.3([7])$. A function $f: \mathbb{T} \rightarrow \mathbb{R}$ is called rd-continuous provided it is continuous at every right-dense point $t \in \mathbb{T}$ and its left-sided limits exist, and is finite at every left-dense point $t \in \mathbb{T}$. The set of rd-continuous functions $f: \mathbb{T} \rightarrow \mathbb{R}$ will be denoted by

$$
C_{r d}=C_{r d}(\mathbb{T})=C_{r d}(\mathbb{T}, \mathbb{R}) .
$$

Definition $2.4([7])$. For $f: \mathbb{T} \rightarrow \mathbb{R}$, we define $f^{\Delta}(t)$ to be the number (if it exists) with the property that for any given $\varepsilon>0$, there exists a neighborhood $U$ of $t$ such that

$$
\left|(f(\sigma(t))-f(s))-f^{\Delta}(t)(\sigma(t)-s)\right|<\varepsilon|\sigma(t)-s|, \quad \text { for all } s \in U .
$$

The function $f^{\Delta}: \mathbb{T}^{k} \rightarrow \mathbb{R}$ is called the delta (or Hilger) derivative of $f$ on $\mathbb{T}^{k}$.

Definition $2.5([7])$. A function $p: \mathbb{T} \rightarrow \mathbb{R}$ is called regressive provided $1+\mu(t) p(t) \neq$ 0 for all $t \in \mathbb{T}$. The set of all regressive and rd-continuous functions $p: \mathbb{T} \rightarrow \mathbb{R}$ will be denoted by $\mathcal{R}=\mathcal{R}(\mathbb{T}, \mathbb{R})$. We define the set $\mathcal{R}^{+}$of all positively regressive elements of $\mathcal{R}$ by

$$
\mathcal{R}^{+}=\mathcal{R}^{+}(\mathbb{T}, \mathbb{R})=\{p \in \mathcal{R}: 1+\mu(t) p(t)>0, \text { for all } t \in \mathbb{T}\} .
$$

Definition $2.6([7])$. Let $p \in \mathcal{R}$, then the generalized exponential function $e_{p}$ is defined as the unique solution of the initial value problem

$$
x^{\Delta}(t)=p(t) x(t), x(s)=1, \quad \text { where } s \in \mathbb{T} .
$$

An explicit formula for $e_{p}(t, s)$ is given by

with

$$
e_{p}(t, s)=\exp \left(\int_{s}^{t} \xi_{\mu(v)}(p(v)) \Delta v\right), \quad \text { for all } s, t \in \mathbb{T},
$$

$$
\xi_{h}(v)= \begin{cases}\frac{\log (1+h v)}{h}, & \text { if } h \neq 0 \\ v, & \text { if } h=0\end{cases}
$$

where log is the principal logarithm function.

Lemma 2.1 ([7]). Let $p, q \in \mathcal{R}$. Then

(i) $e_{0}(t, s) \equiv 1$ and $e_{p}(t, t) \equiv 1$;

(ii) $e_{p}(\sigma(t), s)=(1+\mu(t) p(t)) e_{p}(t, s)$; 
(iii) $\frac{1}{e_{p}(t, s)}=e_{\ominus p}(t, s)$, where $\ominus p(t)=-\frac{p(t)}{1+\mu(t) p(t)}$;

(iv) $e_{p}(t, s)=\frac{1}{e_{p}(s, t)}=e_{\ominus p}(s, t)$;

(v) $e_{p}(t, s) e_{p}(s, r)=e_{p}(t, r)$;

(vi) $\left(\frac{1}{e_{p}(\cdot, s)}\right)^{\Delta}=-\frac{p(t)}{e_{p}^{\sigma}(\cdot, s)}$.

Lemma 2.2 ([1]). If $p \in \mathcal{R}^{+}$, then

$$
0<e_{p}(t, s) \leq \exp \left(\int_{s}^{t} p(v) \Delta v\right), \quad \text { for all } t \in \mathbb{T} .
$$

We end this section by stating the fixed point theorems that we employ to help us show the existence, uniqueness and stability of periodic solutions to $(1.1)$ (see $[9,16]$ ).

Theorem 2.1 (Contraction Mapping Principle). Let $(\chi, \rho)$ a complete metric space and let $\mathcal{P}: \chi \rightarrow \chi$. If there is a constant $\alpha<1$ such that for any $x, y \in \chi$ we have

$$
\rho(\mathcal{P} x, \mathcal{P} y) \leq \alpha \rho(x, y),
$$

then there is one and only one point $z \in \chi$ with $\mathcal{P} z=z$.

Theorem 2.2 (Krasnoselskii). Let $\mathcal{M}$ be a closed bounded convex nonempty subset of a Banach space $(\chi,\|\|$.$) . Suppose that \mathcal{A}$ and $\mathcal{B}$ map $\mathcal{M}$ into $\chi$ such that

(i) $\mathcal{A}$ is compact and continuous;

(ii) $\mathcal{B}$ is a contraction mapping;

(iii) $x, y \in \mathcal{M}$, implies $\mathcal{A} x+\mathcal{B} y \in \mathcal{M}$.

Then there exists $z \in \mathcal{M}$ with $z=\mathcal{A} z+\mathcal{B} z$.

\section{Existence and Uniqueness of Periodic Solutions}

Let $T>0, T \in \mathbb{T}$ be fixed and if $\mathbb{T} \neq \mathbb{R}, T=n \omega$ for some $n \in \mathbb{N}$. By the notation $[a, b]$ we mean

$$
[a, b]=\{t \in \mathbb{T}, a \leq t \leq b\},
$$

unless otherwise specified. The intervals $[a, b),(a, b]$ and $(a, b)$ are defined similarly.

Define $C_{T}=\{\varphi \in C(\mathbb{T}, \mathbb{R}): \varphi(t+T)=\varphi(t)\}$ where $C(\mathbb{T}, \mathbb{R})$ is the space of all real-valued rd-continuous functions. Then $\left(C_{T},\|\|.\right)$ is a Banach space when it is endowed with the supremum norm

$$
\|\varphi\|=\max _{t \in[0, T]}|\varphi(t)| .
$$

We will need the following lemma whose proof can be found in [11].

Lemma 3.1. Let $x \in C_{T}$. Then $\left\|x^{\sigma}\right\|=\|x \circ \sigma\|$ exists and $\left\|x^{\sigma}\right\|=\|x\|$.

In this paper we assume that $a \in \mathcal{R}^{+}, a(t)>0$ for all $t \in \mathbb{T}$ and

$$
a(t+T)=a(t), \quad p(t+T)=p(t), \quad(i d-\tau)(t+T)=(i d-\tau)(t),
$$

with $\tau(t) \geq \tau^{*}>0$ and

$$
e_{a}(T, 0)>1
$$


The functions $g(x), h(x, y)$ are also globally Lipschitz continuous in $x$ and in $x$ and $y$, respectively. That, there are positive constants $k_{1}, k_{2}$ and $k_{3}$ such that

$$
|g(x)-g(y)| \leq k_{1}\|x-y\| \text { and } k_{1}<1
$$

and

$$
|h(x, y)-h(z, w)| \leq k_{2}\|x-z\|+k_{3}\|y-w\| .
$$

Lemma 3.2. Suppose (3.1) and (3.2) hold. If $u \in C_{T}$, then $u$ is a solution of (1.1) if and only if

$$
\begin{aligned}
u(t)= & g(u(t-\tau(t))) \\
& +\gamma \int_{t}^{t+T}\left[p(s)-2 a(s) g\left(u^{\sigma}(s-\tau(s))\right)-h(u(s), u(s-\tau(s)))\right] e_{\ominus a}(t, s) \Delta s,
\end{aligned}
$$

where

$$
\gamma=\left(e_{a}(T, 0)-1\right)^{-1}
$$

Proof. Let $u \in C_{T}$ be a solution of (1.1). Multiply both sides of (1.1) by $e_{a}(t, 0)$ and then integrate from $t$ to $t+T$, to obtain

$$
\begin{aligned}
& \int_{t}^{t+T}\left[(u(s)-g(u(s-\tau(s))))^{\Delta} e_{a}(s, 0)\right] \Delta s \\
= & -\int_{t}^{t+T} a(s)\left[u^{\sigma}(s)-g\left(u^{\sigma}(s-\tau(s))\right] e_{a}(s, 0) \Delta s\right. \\
& +\int_{t}^{t+T}\left[p(s)-2 a(s) g\left(u^{\sigma}(s-\tau(s))-h(u(s), u(s-\tau(s)))\right)\right] e_{a}(s, 0) \Delta s .
\end{aligned}
$$

Performing an integration by part, we obtain

$$
\begin{aligned}
& {[u(t)-g(u(t-\tau(t)))] e_{a}(t, 0)\left(e_{a}(T, 0)-1\right) } \\
& -\int_{t}^{t+T} a(s)\left[u^{\sigma}(s)-g\left(u^{\sigma}(s-\tau(s))\right)\right] e_{a}(s, 0) \Delta s \\
= & -\int_{t}^{t+T} a(s)\left[u^{\sigma}(s)-g\left(u^{\sigma}(s-\tau(s))\right] e_{a}(s, 0) \Delta s\right. \\
& +\int_{t}^{t+T}\left[p(s)-2 a(s) g\left(u^{\sigma}(s-\tau(s))-h(u(s), u(s-\tau(s)))\right)\right] e_{a}(s, 0) \Delta s .
\end{aligned}
$$

By dividing both sides of the above equation by $e_{a}(t, 0)\left(e_{a}(T, 0)-1\right)$, we arrive at

$$
\begin{aligned}
u(t)= & g(u(t-\tau(t)))+\left(e_{a}(T, 0)-1\right)^{-1} \\
& \times \int_{t}^{t+T}\left[p(s)-2 a(s) g\left(u^{\sigma}(s-\tau(s))\right)-h(u(s), u(t-\tau(s)))\right] e_{\ominus a}(t, s) \Delta s .
\end{aligned}
$$

The converse implication is easily obtained and the proof is complete.

By applying Theorems 2.1 and 2.2, we obtain in this Section the existence and the uniqueness of periodic solution of (1.1). So, let a Banach space $\left(C_{T},\|\|.\right)$, a closed bounded convex subset of $C_{T}$,

$$
\mathcal{M}=\left\{\varphi \in C_{T},\|\varphi\| \leq L\right\},
$$


with $L>0$, and by the Lemma 3.2, we define the mapping $\mathcal{P}$ given by $(\mathcal{P} \varphi)(t)=g(\varphi(t-\tau(t)))$

$$
+\gamma \int_{t}^{t+T}\left[p(s)-2 a(s) g\left(\varphi^{\sigma}(s-\tau(s))\right)-h(\varphi(s), \varphi(s-\tau(s)))\right] e_{\ominus a}(t, s) \Delta s .
$$

Therefore, we express (3.7) as

$$
\mathcal{P} \varphi=\mathcal{A} \varphi+\mathcal{B} \varphi
$$

where $\mathcal{A}$ and $\mathcal{B}$ are given by

$$
\begin{aligned}
& (\mathcal{A} \varphi)(t) \\
(3.8)= & \gamma \int_{t}^{t+T}\left[p(s)-2 a(s) g\left(\varphi^{\sigma}(s-\tau(s))\right)-h(\varphi(s), \varphi(s-\tau(s)))\right] e_{\ominus a}(t, s) \Delta s
\end{aligned}
$$

and

$$
(\mathcal{B} \varphi)(t)=g(\varphi(t-\tau(t))) .
$$

Since $\varphi \in C_{T}$ and (3.1) holds, we have for any $\varphi \in \mathcal{M}$

$$
\begin{aligned}
& (\mathcal{A} \varphi)(t+T) \\
= & \gamma \int_{t+T}^{t+T+T}\left[p(s)-2 a(s) g\left(\varphi^{\sigma}(s-\tau(s))\right)-h(\varphi(s), \varphi(s-\tau(s)))\right] e_{\ominus a}(t+T, s) \Delta s \\
= & \gamma \int_{t}^{t+T}\left[p(s+T)-2 a(s+T) g\left(\varphi^{\sigma}(s+T-\tau(s+T))\right)\right. \\
& -h(\varphi(s+T), \varphi(s+T-\tau(s+T)))] e_{\ominus a}(t+T, s+T) \Delta s \\
= & (\mathcal{A} \varphi)(t),
\end{aligned}
$$

and

$$
(\mathcal{B} \varphi)(t+T)=g(\varphi(t+T-\tau(t+T)))=g(\varphi(t-\tau(t)))=(\mathcal{B} \varphi)(t) .
$$

Then

$$
\mathcal{A M}, \mathcal{B \mathcal { M }} \subset C_{T}
$$

Theorem 3.1. Assume that (3.1)-(3.4) hold. Let a constant $L>0$ defined in $\mathcal{M}$ such that

$$
k_{1} L+|g(0)|+\gamma \beta T\left(\mu+2 \lambda k_{1} L+|g(0)|+k_{2} L+k_{3} L+|h(0,0)|\right) \leq L,
$$

where

$$
\beta=e_{a}(T, 0), \quad \lambda=\sup _{t \in[0, T]}\{a(t)\}, \quad \mu=\sup _{t \in[0, T]}|p(t)| .
$$

Then (1.1) has a T-periodic solution. 
Proof. First, let $\mathcal{A}$ defined by (3.8), we show that $\mathcal{A}$ is continuous in the supremum norm and the image of $\mathcal{A}$ is contained in a compact set. Let $\varphi_{n} \in \mathcal{M}$ where $n$ is a positive integer such that $\varphi_{n} \rightarrow \varphi$ as $n \rightarrow \infty$. Then

$$
\begin{aligned}
& \left|\left(\mathcal{A} \varphi_{n}\right)(t)-(\mathcal{A} \varphi)(t)\right| \\
\leq & 2 \gamma \int_{t}^{t+T} a(s)\left|g\left(\varphi_{n}^{\sigma}(s-\tau(s))\right)-g\left(\varphi^{\sigma}(s-\tau(s))\right)\right| e_{\ominus a}(t, s) \Delta s \\
& +\gamma \int_{t}^{t+T}\left|h\left(\varphi_{n}(s), \varphi_{n}(s-\tau(s))\right)-h(\varphi(s), \varphi(s-\tau(s)))\right| e_{\ominus a}(t, s) \Delta s .
\end{aligned}
$$

Since $g$ and $h$ are continuous, the dominated convergence theorem implies,

$$
\lim _{n \rightarrow \infty}\left|\left(\mathcal{A} \varphi_{n}\right)(t)-(\mathcal{A} \varphi)(t)\right|=0,
$$

then $\mathcal{A}$ is continuous. Now, by (3.3) and (3.4), we obtain

$$
\begin{aligned}
|g(y)| & \leq k_{1}|y|+|g(0)|, \\
|h(x, y)| & \leq k_{2}|x|+k_{3}|y|+|h(0,0)| .
\end{aligned}
$$

Then, let $\varphi_{n} \in \mathcal{M}$ where $n$ is a positive integer, we have

$$
\begin{aligned}
& \left|\left(\mathcal{A} \varphi_{n}\right)(t)\right| \\
\leq & \gamma \int_{t}^{t+T}\left[|p(s)|+2 a(s)\left|g\left(\varphi_{n}^{\sigma}(s-\tau(s))\right)\right|+\left|h\left(\varphi_{n}(s), \varphi_{n}(s-\tau(s))\right)\right|\right] e_{\ominus a}(t, s) \Delta s \\
\leq & \gamma \int_{t}^{t+T}\left[p(s)+2 a(s)\left(k_{1}\left\|\varphi_{n}^{\sigma}\right\|+|g(0)|\right)+k_{2}\left\|\varphi_{n}\right\|+k_{3}\left\|\varphi_{n}\right\|+|h(0,0)|\right] e_{\ominus a}(t, s) \Delta s \\
\leq & \gamma \beta T\left(\mu+2 \lambda\left(k_{1} L+|g(0)|\right)+k_{2} L+k_{3} L+|h(0,0)|\right) \leq L,
\end{aligned}
$$

by (3.11). Next, we calculate $\left(\mathcal{A} \varphi_{n}\right)^{\Delta}(t)$ and show that it is uniformly bounded. By making use of (3.1) we obtain by taking the derivative in (3.8) that

$$
\left(\mathcal{A} \varphi_{n}\right)^{\Delta}(t)=-a(t)\left(\mathcal{A} \varphi_{n}\right)^{\sigma}(t)+p(t)-2 a(t) g\left(\varphi_{n}^{\sigma}(t-\tau(t))\right)-h\left(\varphi_{n}(t), \varphi_{n}(t-\tau(t))\right) .
$$

Then, by (3.4) and (3.11) we have

$$
\left|\left(\mathcal{A} \varphi_{n}\right)^{\Delta}(t)\right| \leq \lambda L+\mu+2 \lambda\left(k_{1} L+|g(0)|\right)+k_{2} L+k_{3} L+|h(0,0)|=Q .
$$

Thus the sequence $\left(\mathcal{A} \varphi_{n}\right)$ is uniformly bounded and equicontinuous. Hence, by AscoliArzela's theorem $\mathcal{A} \mathcal{M}$ is compact.

Second, let $\mathcal{B}$ be defined by (3.9). Then for $\varphi_{1}, \varphi_{2} \in \mathcal{M}$ we have by (3.3)

$$
\begin{aligned}
\left|\left(\mathcal{B} \varphi_{1}\right)(t)-\left(\mathcal{B} \varphi_{2}\right)(t)\right| & =\left|g\left(\varphi_{1}(t-\tau(t))\right)-g\left(\varphi_{2}(t-\tau(t))\right)\right| \\
& \leq k_{1}\left\|\varphi_{1}-\varphi_{2}\right\| .
\end{aligned}
$$

Hence, $\mathcal{B}$ is contraction because $k_{1}<1$. 
Finally, we show that if $\varphi, \phi \in \mathcal{M}$, then $\|\mathcal{A} \varphi+\mathcal{B} \phi\| \leq L$. Let $\varphi, \phi \in \mathcal{M}$ with $\|\varphi\|,\|\phi\| \leq L$, then

$$
\begin{aligned}
& \|\mathcal{A} \varphi+\mathcal{B} \phi\| \leq k_{1}\|\phi\|+|g(0)| \\
& +\gamma \int_{t}^{t+T}\left[p(s)+2 a(s)\left(k_{1}\left\|\varphi^{\sigma}\right\|+|g(0)|\right)+k_{2}\|\varphi\|+k_{3}\|\varphi\|+|h(0,0)|\right] e_{\ominus a}(t, s) \Delta s \\
\leq & k_{1} L+|g(0)|+\gamma \beta T\left(\mu+2 \lambda\left(k_{1} L+|g(0)|\right)+k_{2} L+k_{3} L+|h(0,0)|\right) \leq L,
\end{aligned}
$$

by (3.11). Clearly, all the hypotheses of the Krasnoselskii's theorem are satisfied. Thus there exists a fixed point $z \in \mathcal{M}$ such that $z=\mathcal{A} z+\mathcal{B} z$. By Lemma 3.2 this fixed point is a solution of (1.1). Hence, (1.1) has a $T$-periodic solution.

Theorem 3.2. Suppose (3.1)-(3.4) hold. If

$$
k_{1}+\gamma \beta T\left(2 \lambda k_{1}+k_{2}+k_{3}\right)<1,
$$

then (1.1) has a unique T-periodic solution.

Proof. Let the mapping $\mathcal{P}$ be given by (3.7). For any $\varphi_{1}, \varphi_{2} \in C_{T}$, we have

$$
\begin{aligned}
& \left|\left(\mathcal{P} \varphi_{1}\right)(t)-\left(\mathcal{P} \varphi_{2}\right)(t)\right| \\
\leq & \left|g\left(\varphi_{1}(t-\tau(t))\right)-g\left(\varphi_{2}(t-\tau(t))\right)\right| \\
& +2 \gamma \int_{t}^{t+T} a(s)\left|g\left(\varphi_{1}^{\sigma}(s-\tau(s))\right)-g\left(\varphi_{2}^{\sigma}(s-\tau(s))\right)\right| e_{\ominus a}(t, s) \Delta s \\
& +\gamma \int_{t}^{t+T}\left|h\left(\varphi_{1}(s), \varphi_{1}(s-\tau(s))\right)-h\left(\varphi_{2}(s), \varphi_{2}(s-\tau(s))\right)\right| e_{\ominus a}(t, s) \Delta s \\
\leq & k_{1}\left\|\varphi_{1}-\varphi_{2}\right\|+\gamma \int_{t}^{t+T}\left(2 \lambda k_{1}+k_{2}+k_{3}\right)\left\|\varphi_{1}-\varphi_{2}\right\| e_{\ominus a}(t, s) \Delta s \\
\leq & {\left[k_{1}+\gamma \beta T\left(2 \lambda k_{1}+k_{2}+k_{3}\right)\right]\left\|\varphi_{1}-\varphi_{2}\right\| . }
\end{aligned}
$$

Since (3.12) hold, the contraction mapping principle completes the proof.

Corollary 3.1. Suppose (3.1)-(3.4) hold and let $\beta, \lambda$ and $\mu$ be constants defined in Theorem 3.1. Let $\mathcal{M}$ defined by (3.6). Suppose there are positive constants $k_{1}^{*}, k_{2}^{*}$ and $k_{3}^{*}$ such that for any $x, y, z, w \in \mathcal{M}$, we have

$$
\begin{gathered}
|g(x)-g(y)| \leq k_{1}^{*}\|x-y\| \text { and } k_{1}^{*}<1, \\
|h(x, y)-h(z, w)| \leq k_{2}^{*}\|x-z\|+k_{3}^{*}\|y-w\|
\end{gathered}
$$

and

$$
k_{1}^{*} L+|g(0)|+\gamma \beta T\left(\mu+2 \lambda\left(k_{1}^{*} L+|g(0)|\right)+k_{2}^{*} L+k_{3}^{*} L+|h(0,0)|\right) \leq L .
$$

Then (1.1) has a T-periodic solution in $\mathcal{M}$. Moreover, if

$$
k_{1}^{*}+\gamma \beta T\left(2 \lambda k_{1}^{*}+k_{2}^{*}+k_{3}^{*}\right)<1,
$$

then (1.1) has a unique T-periodic solution in $\mathcal{M}$. 
Proof. Let the mapping $\mathcal{P}$ defined by (3.7). Then the proof follow immediately from Theorem 3.1 and Theorem 3.2.

Notice that the constants $k_{1}^{*}, k_{2}^{*}$ and $k_{3}^{*}$ may depend on $L$.

\section{Existence of Positive Periodic Solutions}

It is for sure that existence of positive solutions is important for many applied problems. In this Section, by applying the Krasnoselskii's fixed point theorem and some techniques, to establish a set of sufficient conditions which guarantee the existence of positive periodic solutions of (1.1). So, we let $(\chi,\|\|)=.\left(C_{T},\|\cdot\|\right)$ and $\mathcal{M}(E, K)=$ $\left\{\varphi \in C_{T}: E \leq \varphi(t) \leq K\right.$ for all $\left.t \in[0, T]\right\}$, for any $0<E<K$. We assume that, there exist constants $a_{1}, a_{2}, g_{1}$ and $g_{2}$ such that for all $(t,(x, y, z)) \in[0, T] \times[E, K]^{3}$ we have

$$
\begin{gathered}
0 \leq g_{1}, \quad 0 \leq g_{2}<1, \quad-g_{1} y \leq g(y) \leq g_{2} y, \\
0<a_{1} \leq a(t) \leq a_{2}, \\
\left(E+g_{1} K\right) a_{2} \leq p(t)-2 a(t) g(z)-h(x, y) \leq\left(1-g_{2}\right) K a_{1} .
\end{gathered}
$$

Theorem 4.1. Assume that (3.1)-(3.4) and (4.1)-(4.3) hold. Then (1.1) has at least one positive $T$-periodic solution in $\mathcal{M}(E, K)$.

Proof. By Lemma 3.2, it is obvious that (1.1) has a solution $\varphi$ if and only if $\mathcal{P} \varphi=\varphi$ has a solution $\varphi$. Let $\mathcal{A}, \mathcal{B}$ defined by (3.8), (3.9) respectively. A change of variable $t \mapsto t+T$ in (3.8) and (3.9) show that for any $\varphi \in \mathcal{M}(E, K)$ and $t \in \mathbb{R}$

$$
\mathcal{A}(\mathcal{M}(E, K)) \subseteq C_{T}, \quad \mathcal{B}(\mathcal{M}(E, K)) \subseteq C_{T} .
$$

Arguing as in the Theorem 3.1, the operator $\mathcal{A}$ is continuous. Next, we claim that $\mathcal{A}$ is compact. It is sufficient to show that $\mathcal{A}(\mathcal{M}(E, K))$ is uniformly bounded and equicontinuous in $[0, T]$. Notice that (4.2) and (4.3) ensure that

$$
\begin{aligned}
& \|\mathcal{A} \varphi\| \\
\leq & \sup _{t \in[0, T]}\left|\gamma \int_{t}^{t+T}\left[p(s)-2 a(s) g\left(\varphi^{\sigma}(s-\tau(s))\right)-h(\varphi(s), \varphi(s-\tau(s)))\right] e_{\ominus a}(t, s) \Delta s\right| \\
\leq & \left(1-g_{2}\right) K \gamma a_{1} \sup _{t \in[0, T]} \int_{t}^{t+T} e_{\ominus a}(t, s) \Delta s \\
\leq & \left(1-g_{2}\right) K, \quad \text { for all } \varphi \in[E, K]
\end{aligned}
$$

and

$$
\begin{aligned}
& \left|(\mathcal{A} \varphi)^{\Delta}(t)\right| \\
\leq & a(t)\left|(\mathcal{A} \varphi)^{\sigma}(t)\right|+\left|p(t)-2 a(t) g\left(\varphi^{\sigma}(t-\tau(t))\right)-h(\varphi(t), \varphi(s-\tau(t)))\right| \\
\leq & a_{2}\left(1-g_{1}\right) K+\left(1-g_{1}\right) a_{1} K \\
= & \left(a_{2}+a_{1}\right)\left(1-g_{1}\right) k, \quad \text { for all }(t, \varphi) \in[0, T] \times[E, K],
\end{aligned}
$$


which give that $\mathcal{A}(\mathcal{M}(E, K))$ is uniformly bounded and equicontinuous in $[0, T]$. Hence by Ascoli-Arzela's theorem $\mathcal{A}$ is compact. Next, let $\mathcal{B}$ defined by (3.9), for all $\varphi_{1}, \varphi_{2} \in \mathcal{M}(E, K)$ and $t \in \mathbb{R}$, we obtain by (3.3)

$$
\left\|\mathcal{B} \varphi_{1}-\mathcal{B} \varphi_{2}\right\| \leq k_{1}\left\|\varphi_{1}-\varphi_{2}\right\|
$$

Thus $\mathcal{B}$ is a contraction. Moreover, by (4.1)-(4.3), we infer that for all $\varphi, \phi \in \mathcal{M}(E, K)$ and $t \in \mathbb{R}$

$$
\begin{aligned}
& (\mathcal{A} \varphi)(t)+(\mathcal{B} \phi)(t) \\
= & g(\phi(t-\tau(t))) \\
& +\gamma \int_{t}^{t+T}\left[p(s)-2 a(s) g\left(\varphi^{\sigma}(s-\tau(s))\right)-h(\varphi(s), \varphi(s-\tau(s)))\right] e_{\ominus a}(t, s) \Delta s \\
\leq & g_{2} K+\left(1-g_{2}\right) K \gamma \int_{t}^{t+T} a(s) e_{\ominus a}(t, s) \Delta s=K .
\end{aligned}
$$

On the other hand,

$$
\begin{aligned}
& (\mathcal{A} \varphi)(t)+(\mathcal{B} \phi)(t) \\
= & g(\phi(t-\tau(t))) \\
& +\gamma \int_{t}^{t+T}\left[p(s)-2 a(s) g\left(\varphi^{\sigma}(s-\tau(s))\right)-h(\varphi(s), \varphi(s-\tau(s)))\right] e_{\ominus a}(t, s) \Delta s \\
\geq & -g_{1} K+\left(E+g_{1} K\right) \gamma \int_{t}^{t+T} a(s) e_{\ominus a}(t, s) \Delta s=E,
\end{aligned}
$$

which imply that

$$
\mathcal{A} \varphi+\mathcal{B} \phi \in \mathcal{M}(E, K), \quad \text { for all } \varphi, \phi \in \mathcal{M}(E, K) \text { and } t \in \mathbb{R} .
$$

Clearly, all the hypotheses of the Krasnoselskii's theorem are satisfied. Thus there exists a fixed point $z \in \mathcal{M}(E, K)$ such that $z=\mathcal{A} z+\mathcal{B} z$. By Lemma 3.2 this fixed point is a solution of (1.1). Hence, (1.1) has a positive $T$-periodic solution. This completes the proof.

Theorem 4.2. Assume that (3.1)-(3.4) hold. Suppose that there exist constants E, $K, a_{1}, a_{2}, g_{1}, g_{2}$ and $t_{0} \in[0, T]$ satisfying (4.1)-(4.3) with

$$
0 \leq E<K
$$

and either

$$
\left(E+g_{1} K\right) a_{2}<p\left(t_{0}\right)-2 a\left(t_{0}\right) g(z)-h(x, y), \quad \text { for all } x, y, z \in[E, K],
$$

or

$$
a\left(t_{0}\right)<a_{2} .
$$

Then (1.1) has at least one positive T-periodic solution in $\mathcal{M}(E, K)$, with $E<u \leq K$ for each $t \in[0, T]$. 
Proof. As in the proof of Theorem 4.1, we conclude similarly that (1.1) has an $T$ periodic solution $u \in \mathcal{M}(E, K)$. Now we assert that $u(t)>E$ for all $t \in[0, T]$. Otherwise, there exists $t^{*} \in[0, T]$ satisfying $u\left(t^{*}\right)=E$. In view of (3.5), (3.7), (4.1) and (4.6), we have

$$
\begin{aligned}
E= & g\left(u\left(t^{*}-\tau\left(t^{*}\right)\right)\right) \\
& +\gamma \int_{t^{*}}^{t^{*}+T}\left[p(s)-2 a(s) g\left(u^{\sigma}(s-\tau(s))\right)-h(u(s), u(s-\tau(s)))\right] e_{\ominus a}\left(t^{*}, s\right) \Delta s \\
\geq & \gamma \int_{t^{*}}^{t^{*}+T}\left[p(s)-2 a(s) g\left(u^{\sigma}(s-\tau(s))\right)-h(u(s), u(s-\tau(s)))\right] e_{\ominus a}\left(t^{*}, s\right) \Delta s \\
& -g_{1} K,
\end{aligned}
$$

which implies that

$$
\begin{aligned}
0 \geq & \gamma \int_{t^{*}}^{t^{*}+T}\left[p(s)-2 a(s) g\left(u^{\sigma}(s-\tau(s))\right)-h(u(s), u(s-\tau(s)))\right] e_{\ominus a}\left(t^{*}, s\right) \Delta s \\
& -\left(E+g_{1} K\right) \\
= & \gamma \int_{t^{*}}^{t^{*}+T}\left[p(s)-2 a(s) g\left(u^{\sigma}(s-\tau(s))\right)-h(u(s), u(s-\tau(s)))\right. \\
& \left.-\left(E+g_{1} K\right) a(s)\right] e_{\ominus a}\left(t^{*}, s\right) \Delta s .
\end{aligned}
$$

Assume that (4.7) holds. By means of (4.2), (4.3), (4.7) and the continuity of $h, g, a$, $p, \tau$ and $u$, we get that

$$
\begin{aligned}
& \gamma \int_{t^{*}}^{t^{*}+T} e_{\ominus a}\left(t^{*}, s\right)\left[p(s)-2 a(s) g\left(u^{\sigma}(s-\tau(s))\right)-h(u(s), u(s-\tau(s)))\right. \\
& \left.-\left(E+g_{1} K\right) a(s)\right] \Delta s \\
\geq & \int_{t^{*}}^{t^{*}+T} e_{\ominus a}\left(t^{*}, s\right)\left[p(s)-2 a(s) g\left(u^{\sigma}(s-\tau(s))\right)-h(u(s), u(s-\tau(s)))\right. \\
& \left.-\left(E+g_{1} K\right) a_{2}\right] \Delta s \\
> & 0
\end{aligned}
$$

which contradicts (4.9).

Assume that (4.8) holds. In light of (4.2), (4.3), (4.8) and the continuity of $h, g, a$, $p, \tau$ and $u$, we get that

$$
\begin{aligned}
& \gamma \int_{t^{*}}^{t^{*}+T}\left[p(s)-2 a(s) g\left(u^{\sigma}(s-\tau(s))\right)-h(u(s), u(s-\tau(s)))\right. \\
& \left.-\left(E+g_{1} K\right) a(s)\right] e_{\ominus a}\left(t^{*}, s\right) \Delta s \\
> & \int_{t^{*}}^{t^{*}+T} e_{\ominus a}\left(t^{*}, s\right) \Delta s \int_{t^{*}}^{t^{*}+T} e_{\ominus a}\left(t^{*}, s\right)\left[p(s)-2 a(s) g\left(u^{\sigma}(s-\tau(s))\right)\right. \\
& \left.-h(u(s), u(s-\tau(s)))-\left(E+g_{1} K\right) a_{2}\right] \Delta s \\
> & 0,
\end{aligned}
$$

which contradicts (4.9). This completes the proof. 
Example 4.1. Consider (1.1), where

$$
\begin{gathered}
\mathbb{T}=\mathbb{R}, \quad p(t)=3+\frac{\sin t}{5}, \quad a(t)=1+\frac{\cos t}{4}, \quad \tau(t)=2 \cos ^{2} t, \\
g(x)=-\frac{x \sin x}{20}, \quad \text { for all } x \in \mathbb{R}, \\
h(x, y)=1+\sin ^{2} x+\cos ^{2} y, \quad \text { for all }(x, y) \in \mathbb{R}^{2} .
\end{gathered}
$$

Let $T=2 \pi, K=10, E=1, g_{1}=g_{2}=\frac{1}{20}, a_{1}=\frac{3}{4}, a_{2}=\frac{5}{4}, k_{1}=\frac{11}{20}$. It is easy to see that (3.3), (3.4) hold. Notice that

$$
\begin{aligned}
\left(E+g_{1} K\right) a_{2} & =\frac{15}{8}<\frac{195}{40}=3-\frac{1}{5}+2\left(1-\frac{1}{4}\right) \frac{1}{20}+2 \\
& \leq p(t)-2 a(t) g(z)-h(x, y) \\
& \leq 3+\frac{1}{5}+2 \cdot \frac{5}{4} \cdot \frac{1}{20}+3=\frac{253}{40} \\
& <\frac{285}{40}=\left(1-g_{2}\right) K a_{1}, \quad \text { for all }(t, x, y, z) \in \mathbb{R}^{4} .
\end{aligned}
$$

That is, (4.3) is satisfied. Thus Theorem 4.1 yields that (1.1) has a positive $2 \pi$-periodic solution in $\mathcal{M}(1,10)$.

\section{Stability of Periodic Solutions}

This Section concerned with the stability of a $T$-periodic solution $u^{*}$ of (1.1). Let $v=u-u^{*}$ then (1.1) is transformed as

$$
\begin{aligned}
& (v(t)-G(v(t-\tau(t))))^{\Delta} \\
= & -a(t) v^{\sigma}(t)-a(t) G\left(v^{\sigma}(t-\tau(t))\right)-H(v(t), v(t-\tau(t))),
\end{aligned}
$$

where

$$
G(v(t-\tau(t)))=g\left(u^{*}(t-\tau(t))+v(t-\tau(t))\right)-g\left(u^{*}(t-\tau(t))\right.
$$

and

$$
\begin{aligned}
& H(v(t), v(t-\tau(t))) \\
= & h\left(u^{*}(t)+v(t)\right), u^{*}(t-\tau(t))+v(t-\tau(t))-h\left(u^{*}(t), u^{*}(t-\tau(t))\right) .
\end{aligned}
$$

Clearly, (5.1) has trivial solution $v \equiv 0$, and the conditions (3.3) and (3.4) hold for $G$ and $H$ respectively. To arrive at the Lemma 3.2, as in the proof of this Lemma, multiply both sides of $(5.1)$ by $e_{a}(t, 0)$ and then integrate from 0 to $t$, to obtain

$$
\begin{aligned}
v(t)= & (v(0)-G(v(-\tau(0)))) e_{\ominus a}(t, 0)+G(v(t-\tau(t))) \\
& -\int_{0}^{t}\left[2 a(s) G\left(v^{\sigma}(s-\tau(s))\right)+H(v(s), v(s-\tau(s)))\right] e_{\ominus a}(t, s) \Delta s .
\end{aligned}
$$

Thus, we see that $v$ is a solution of (5.1) if and only if it satisfies (5.2). Assumed initial function

$$
v(t)=\psi(t), \quad t \in\left[m_{0}, 0\right]
$$


with $\psi \in C\left(\left[m_{0}, 0\right], R\right),\left[m_{0}, 0\right]=\{s \leq 0 \mid s=t-\tau(t), t \geq 0\}$. For the stability definition we refer the reader to the book [9].

Define the set $S_{\psi}$ by

$$
S_{\psi}=\left\{\varphi \in C_{T},\|\varphi\| \leq R, \varphi(t)=\psi(t) \text { if } t \in\left[m_{0}, 0\right], \varphi(t) \rightarrow 0 \text { as } t \rightarrow \infty\right\},
$$

for some positive constant $R$. Then, $\left(S_{\psi},\|\|.\right)$ is a complete metric space where $\|\cdot\|$ is the supremum norm.

Theorem 5.1. If (3.1), (3.3), (3.4) and

$$
\begin{aligned}
& e_{\ominus a}(t, 0) \rightarrow 0 \text { as } t \rightarrow \infty, \\
& t-\tau(t) \rightarrow \infty \text { as } t \rightarrow \infty \\
& k_{1}+\int_{0}^{t}\left(2 \lambda k_{1}+k_{2}+k_{3}\right) e_{\ominus a}(t, s) \Delta s \leq \alpha<1,
\end{aligned}
$$

hold. Then every solution $v(t, 0, \psi)$ of (5.1) with small continuous initial function $\psi$, is bounded and asymptotically stable.

Proof. Let the mapping $\mathcal{F}$ defined by $\psi(t)$ if $t \in\left[m_{0}, 0\right]$ and

$$
\begin{aligned}
(\mathcal{F} \varphi)(t)= & (\psi(0)-G(\psi(-\tau(0)))) e_{\ominus a}(t, 0)+G(\varphi(t-\tau(t))) \\
& -\int_{0}^{t}\left[2 a(s) G\left(\varphi^{\sigma}(s-\tau(s))\right)+H(\varphi(s), \varphi(s-\tau(s)))\right] e_{\ominus a}(t, s) \Delta s,
\end{aligned}
$$

if $t \geq 0$. Since $G$ and $H$ are continuous, it is easy to show that $\mathcal{F} \varphi$ is continuous. Let $\psi$ be a small given continuous initial function with $\|\psi\|<\delta(\delta>0)$. Then using the condition (5.6) and the definition of $\mathcal{F}$ in (5.7), we have for $\varphi \in S_{\psi}$

$$
\begin{aligned}
|(\mathcal{F} \varphi)(t)| \leq & |\psi(0)-G(\psi(-\tau(0)))| e_{\ominus a}(t, 0)+k_{1} R \\
& +R \int_{0}^{t}\left(2 \lambda k_{1}+k_{2}+k_{3}\right) e_{\ominus a}(t, s) \Delta s \\
\leq & \left(1+k_{1}\right) \delta+k_{1} R+R \int_{0}^{t}\left(2 \lambda k_{1}+k_{2}+k_{3}\right) e_{\ominus a}(t, s) \Delta s \\
\leq & \left(1+k_{1}\right) \delta+\alpha R \leq R,
\end{aligned}
$$

which implies $\|\mathcal{F} \varphi\| \leq R$, for the right $\delta$. Next we show that $(\mathcal{F} \varphi)(t) \rightarrow 0$ as $t \rightarrow \infty$. The first term on the right side of (5.7) tends to zero, by condition (5.4). Also, the second term on the right side tends to zero, because of (5.5) and the fact that $\varphi \in S_{\psi}$. Let $\epsilon>0$ be given, then there exists a $t_{1}>0$ such that for $t>t_{1}, \varphi(t-\tau(t))<\epsilon$. By the condition (5.4), there exists a $t_{2}>t_{1}$ such that for $t>t_{2}$ implies that

$$
e_{\ominus a}\left(t, t_{2}\right)<\frac{\epsilon}{\alpha R}
$$


Thus for $t>t_{2}$, we have

$$
\begin{aligned}
& \left|\int_{0}^{t}\left[2 a(s) G\left(\varphi^{\sigma}(s-\tau(s))\right)+H(\varphi(s), \varphi(s-\tau(s)))\right] e_{\ominus a}(t, s) \Delta s\right| \\
\leq & R \int_{0}^{t_{1}}\left(2 \lambda k_{1}+k_{2}+k_{3}\right) e_{\ominus a}(t, s) \Delta s+\epsilon \int_{0}^{t}\left(2 \lambda k_{1}+k_{2}+k_{3}\right) e_{\ominus a}(t, s) \Delta s \\
\leq & R e_{\ominus a}\left(t, t_{2}\right) \int_{0}^{t}\left(2 \lambda k_{1}+k_{2}+k_{3}\right) e_{\ominus a}\left(t_{2}, s\right) \Delta s+\alpha \epsilon \\
\leq & \alpha R e_{\ominus a}\left(t, t_{2}\right) \alpha+\alpha \epsilon<\alpha \epsilon+\epsilon .
\end{aligned}
$$

Hence, $(\mathcal{F} \varphi)(t) \rightarrow 0$ as $t \rightarrow \infty$. It is natural now to prove that $\mathcal{F}$ is contraction under the supremum norm. Let $\varphi_{1}, \varphi_{2} \in S_{\psi}$. Then

$$
\begin{aligned}
& \left|\left(\mathcal{F} \varphi_{1}\right)(t)-\left(\mathcal{F} \varphi_{2}\right)(t)\right| \\
\leq & \left|G\left(\varphi_{1}(t-\tau(t))\right)-G\left(\varphi_{2}(t-\tau(t))\right)\right| \\
& +2 \lambda \int_{0}^{t}\left|G\left(\varphi_{1}^{\sigma}(s-\tau(s))\right)-G\left(\varphi_{2}^{\sigma}(s-\tau(s))\right)\right| e_{\ominus a}(t, s) \Delta s \\
& +\int_{0}^{t}\left|H\left(\varphi_{1}(s), \varphi_{1}(s-\tau(s))\right)-H\left(\varphi_{2}(s), \varphi_{2}(s-\tau(s))\right)\right| e_{\ominus a}(t, s) \Delta s \\
\leq & k_{1}\left\|\varphi_{1}-\varphi_{2}\right\|+\int_{0}^{t}\left(2 \lambda k_{1}+k_{2}+k_{3}\right)\left\|\varphi_{1}-\varphi_{2}\right\| e_{\ominus a}\left(t_{2}, s\right) \Delta s \\
\leq & {\left[k_{1}+\int_{0}^{t}\left(2 \lambda k_{1}+k_{2}+k_{3}\right) e_{\ominus a}\left(t_{2}, s\right) \Delta s\right]\left\|\varphi_{1}-\varphi_{2}\right\| } \\
\leq & \alpha\left\|\varphi_{1}-\varphi_{2}\right\| .
\end{aligned}
$$

Hence, the contraction mapping principle implies, $\mathcal{F}$ has a unique fixed point in $S_{\psi}$ which solves (5.1), bounded and asymptotically stable.

Theorem 5.2. If (3.1), (3.3), (3.4) and (5.6) hold. Then, the zero solution is stable.

Proof. The stability of the zero solution of (5.1) follows simply by replacing $R$ by $\epsilon$ in the above theorem.

Acknowledgements. The authors would like to thank the anonymous referee for his/her valuable comments and good advice.

\section{REFERENCES}

[1] M. Adivar and Y. N. Raffoul, Existence of periodic solutions in totally nonlinear delay dynamic equations, Electron. J. Qual. Theory Differ. Equ. 2009(1) (2009), 1-20.

[2] A. Ardjouni and A. Djoudi, Existence of positive periodic solutions for nonlinear neutral dynamic equations with variable delay on a time scale, Malaya Journal of Matematik 2(1) (2013), 60-67.

[3] A. Ardjouni and A. Djoudi, Existence of periodic solutions for nonlinear neutral dynamic equations with functional delay on a time scale, Acta Univ. Palack. Olomuc. Fac. Rerum Natur. Math. 52(1) (2013), 5-19.

[4] A. Ardjouni and A. Djoudi, Existence of periodic solutions for nonlinear neutral dynamic equations with variable delay on a time scale, Commun. Nonlinear Sci. Numer. Simul. 17 (2012), 3061-3069. 
[5] A. Ardjouni and A Djoudi, Stability in neutral nonlinear dynamic equations on time scale with unbounded delay, Stud. Univ. Babeç-Bolyai Math. 57(4) (2012), 481-496.

[6] A. Ardjouni and A. Djoudi, Periodic solutions in totally nonlinear dynamic equations with functional delay on a time scale, Rend. Semin. Mat. Univ. Politec. Torino 68(4) (2010), 349-359.

[7] M. Bohner and A. Peterson, Dynamic Equations on Time Scales, An Introduction with Applications, Birkhäuser, Boston, 2001.

[8] M. Bohner and A. Peterson, Advances in Dynamic Equations on Time Scales, Birkhäuser, Boston, 2003.

[9] T. A. Burton, Stability by Fixed Point Theory for Functional Differential Equations, Dover Publications, New York, 2006.

[10] S. Hilger, Ein Masskettenkalkül mit Anwendung auf Zentrumsmanningfaltigkeiten, $\mathrm{PhD}$ thesis, Universität Würzburg, 1988.

[11] E. R. Kaufmann and Y. N. Raffoul, Periodic solutions for a neutral nonlinear dynamical equation on a time scale, J. Math. Anal. Appl. 319 (2006) 315-325.

[12] E. R. Kaufmann and Y. N. Raffoul, Periodicity and stability in neutral nonlinear dynamic equation with functional delay on a time scale, Electron. J. Differential Equations 2007(27) (2007), 1-12.

[13] V. Lakshmikantham, S. Sivasundaram and B. Kaymarkcalan, Dynamic Systems on Measure Chains, Kluwer Academic Publishers, Dordrecht, 1996.

[14] M. B. Mesmouli, A. Ardjouni and A. Djoudi, Existence and stability of periodic solutions for nonlinear neutral differential equations with variable delay using fixed point technique, Acta Univ. Palack. Olomuc. Fac. Rerum Natur. Math. 54(1) (2015), 95-108.

[15] R. Olach, Positive periodic solutions of delay differential equations, Appl. Math. Lett. 26 (2013), 1141-1145.

[16] D. R. Smart, Fixed Points Theorems, Cambridge University Press, Cambridge, UK, 1980.

[17] Y. Yuan and Z. Guo, On the existence and stability of periodic solutions for a nonlinear neutral functional differential equation, Abstr. Appl. Anal. 2013 (2013), 1-8.

${ }^{1}$ Department of MATHEMATiCs,

UNIVERSITY OF ANNABA,

P.O. Box 12, Annaba, Algeria

Email address: fyb1500@gmail.com

Email address: adjoudi@yahoo.com

${ }^{2}$ Department of Mathematics And Informatics, University of Souk Ahras,

P.O. Box 1553, Souk Ahras, Algeria

Email address: abd_ardjouni@yahoo.fr 